\title{
A Novel Estimation Approach of Pressure Gradient and Haemodynamic Stresses as Indicators of Pathological Aortic Flow Using Subvoxel Modelling
}

\author{
Pascal Corso, George Giannakopoulos, Utku Gülan, Christos Emmanouil Frouzakis, and Markus Holzner
}

\begin{abstract}
Objective: The flow downstream from aortic stenoses is characterised by the onset of shear-induced turbulence that leads to irreversible pressure losses. These extra losses represent an increased resistance that impacts cardiac efficiency. A novel approach is suggested in this study to accurately evaluate the pressure gradient profile along the aorta centreline using modelling of haemodynamic stress at scales that are smaller than the typical resolution achieved in experiments. Methods: We use benchmark data obtained from direct numerical simulation (DNS) along with results from in silico and in vitro threedimensional particle tracking velocimetry (3D-PTV) at three voxel sizes, namely $750 \mu \mathrm{m}, 1 \mathrm{~mm}$ and $1.5 \mathrm{~mm}$. A differential equation is derived for the pressure gradient, and the subvoxelscale (SVS) stresses are closed using the Smagorinsky and a new refined model. Model constants are optimised using DNS and in silico PTV data and validated based on pulsatile in vitro 3D-PTV data and pressure catheter measurements. Results: The Smagorinsky-based model was found to be more accurate for SVS stress estimation but also more sensitive to errors especially at lower resolution, whereas the new model was found to more accurately estimate the projected pressure gradient even for larger voxel size of $1.5 \mathrm{~mm}$ albeit at the cost of increased sensitivity at this voxel size. A comparison with other methods in the literature shows that the new approach applied to in vitro PTV measurements estimates the irreversible pressure drop by decreasing the errors by at least $20 \%$. Conclusion: Our novel approach based on the modelling of subvoxel stress offers a validated and more accurate way to estimate pressure gradient, irreversible pressure loss and SVS stress. Significance: We anticipate that the approach may potentially be applied to image-based in vivo, in vitro 4D flow data or in silico data with limited spatial resolution to assess pressure loss and SVS stresses in disturbed aortic blood flow.
\end{abstract}

Index Terms-aortic stenosis, turbulence, pressure drop, SVS stress, DNS, 3D-PTV. high transvalvular pressure difference results in an increased load on the left ventricle that can lead to hypertrophy and dysfunction thereof. The prevalence of AS increases with age and can be as high as $4.6 \%$ in people older than 75 years of age [27]. Severe AS can also cause irreversible damage to blood cells due to the augmented stress red cells and platelets experience [26].

Non-invasive Doppler echocardiography is the recommended clinical standard to assess AS severity [3]. Guidelines suggest to consider the peak transvalvular velocity, the mean transvalvular pressure gradient calculated using a simplified Bernoulli equation and the continuity-equation-derived aortic valve area as the three primary haemodynamic parameters for the assessment of AS severity [3]. Such commonly used parameters for pressure drop estimation are based on strong haemodynamic approximations and do not take into account the interaction between valve, left ventricle and peripheral vasculature ([13], [14], [22]). In fact, these indicators give only partial information about the detrimental effects on the left ventricle linked to the pressure loss caused by the malfunctioning aortic valve [22].

It has been reported that the transvalvular velocity peak due to the reduction in cross-sectional area is not sufficient to accurately estimate the additional pressure loss as it is dependent on the flow conditions and a the threshold value on velocity to evaluate stenosis severity would have to be then patient dependent [3].

The mean transvalvular pressure drop is calculated by means of a modified or simplified Bernoulli law using only the velocity averaged over the systolic phase in the vicinity of the valve. This does not take into account the energy loss due to the turbulent stresses brought about downstream from the valve nor the energy loss across the stenosis orifice [28]. As a result, for severe stenosis, the actual pressure drop is underestimated when the simplified Bernoulli relation is used. The degree of underestimation found in the literature varies between 5\% to 50\% depending on the orifice area as well as on the flow unsteadiness ([12], [15], [28]). In addition, it has been shown that for mild to moderate stenosis with gradual distal widening, the pressure drop localised at the valve calculated with the simplified Bernoulli approximation overestimates the actual pressure loss measured further downstream due to the recovered pressure related to the decrease in velocity [3], [22]. Other more complete models based on Bernoulli's law have been suggested and take into account energy losses across the stenotic nozzle and downstream of the stenotic valve
2

3

4

This document is the accepted manuscript version of the following article:

Corso, P., Giannakopoulos, G., Gulan, U., Frouzakis, C. E., \& Holzner, M. (2020). A novel estimation approach of pressure gradient and haemodynamic stresses as indicators of pathological aortic flow using subvoxel modelling. IEEE Transactions on Biomedical Engineering. https://doi.org/10.1109/ 
along with the unsteady effects of flow acceleration during systole ([13], [28]). Many of these models use as independent variable the effective orifice area (EOA) whose evaluation based for instance on the continuity equation is a source of inaccuracies and uncertainties [4]. Hatoum et al. [17] have recently proposed a model that instead of the estimate of EOA requires empirical constants which, however, might not be suitable for a wide range of cases. The energy loss index is a complementary indicator that accounts for irreversible energy loss occurring in the aorta. The index is derived from Bernoulli's law and the integrated linear momentum equation projected on the flow axis obtained under various assumptions, i.e. uniform velocity profile, negligible acceleration term and the estimation of EOA [13].

Finally, it is important to note that in addition to the assumptions made in order to formulate the aforementioned indicators, measurement errors introduced in Doppler echocardiography can be significant, due to the misalignment of the ultrasound beam with respect to the flow direction resulting in a too high intercept angle [3].

Although pressure loss evaluation from Doppler echocardiography can be easily implemented on patients in vivo and can provide useful order of magnitude estimates of pressure loss within a short acquisition time, in recent years, there has been an effort to indirectly estimate pressure gradient and energy loss by characterising the three-dimensional velocity field using in vivo 4D flow magnetic resonance imaging (MRI) and in vitro particle tracking velocimetry (PTV) [9], [14][16], [19]. This has overcome some of the limitations of Doppler echocardiography estimates. It is still based though on several assumptions and the influence of limited spatial resolution has never been investigated systematically. In fact, 4D flow magnetic resonance imaging (MRI) has recently been employed to characterise turbulence in aortic flow and evaluate irreversible pressure drop [15], [16]. Since pressure cannot be directly measured from MRI measurements, Ha et al. [15] assumed that the rate of work done by the pressure drop in the stenosed aorta is equivalent to the total energy dissipated in the flow with the latter estimated by the integral over the whole aorta volume of the production of turbulent kinetic energy at voxel sizes of 1 to $2 \mathrm{~mm}$. A dedicated MR sequence has then been developed and this sequence has been validated against results obtained from large-eddy simulations to evaluate the six components of the Reynolds stress tensor [15]. The product of this reconstructed tensor with the shear-rate tensor obtained from the MR-based velocity field gives the production of turbulent kinetic energy. Moreover, it has been shown in [14] and [16] that the latter can also be evaluated using a shearscaling argument requiring the use of a model constant and the calculation of the mean shear rate and the diagonal elements of Reynolds stress tensor. The limitations of such an approach based on macroscopic energy balance lie in the applicability of the aforementioned main assumptions to complex and bended aorta geometries, to pulsatile flow conditions and to moving boundary walls.

The approaches summarised above are based on several assumptions to model pressure loss and focus on bulk pressure loss, i.e. the loss over an extended control volume. To date, there is no method available that is capable of predicting the pressure gradient evolution across a given volume of interest downstream of a stenosis. Furthermore, due to limited spatial resolutions in flow measurement techniques such as particle tracking velocimetry or 4D MRI, the evaluation of small-scale stresses owing to turbulence in the flow still requires adequate and validated models.

In this study, a direct numerical simulation of stenotic flow using a spectral element flow solver is used as a benchmark to establish a novel approach based on a rigorously derived differential equation to evaluate pressure gradient along the centreline of stenosed aortas. DNS data are thus used to optimise models for subvoxel-scale stress estimation. In fact, we test the hypothesis that the limited resolution inherent in in vitro and in vivo measurements, e.g. for 3D-PTV or 4D Flow MRI, can be represented by a spatial filter and consequently the under-resolved scales modelled with an LES-type model. A systematic validation of the models for three voxel sizes is performed. This new approach has been established based on the characterisation of the flow in a stenosed aorta geometry from in silico and in vitro three-dimensional particle tracking velocimetry (3D-PTV) data. Finally, we also verify through pressure catheter measurements the applicability of this new approach to pulsatile flow conditions in a compliant aorta model with a different stenosis geometry.

\section{Methodology}

Three methods are used to investigate stenotic flows. The first investigated case concerns the flow downstream of a severe stenosis of a tricuspid aortic valve in a rigid aorta geometry (see Fig. 1). The combination of direct numerical simulation, in silico and in vitro particle tracking velocimetry is applied to that flow case and allows us to develop and optimise two models for subvoxel stress estimation as part of our approach for pressure gradient evaluation. The validation is performed for three different voxel sizes representative of the resolution reached in in vivo 4D Flow MR and in vitro 3D-PTV data, namely, $0.75,1$ and $1.5 \mathrm{~mm}$. The second investigated case deals with the flow after a concentric severe aortic stenosis in the root of a compliant model of the same aorta geometry as that of the first case. A pulsating flow rate is imposed upstream from the stenosis and the approach based upon subvoxel-scale models previously elaborated is validated using in vitro 3DPTV and pressure catheter measurements.

\section{A. Direct Numerical Simulation (DNS)}

The aorta geometry was obtained from an MRI scan and has a diameter at the sinotubuluar junction of $25 \mathrm{~mm}$. A geometrical model of a stenosis was added to the sinotubular extremity. This model represents a severe calcified stenosis of a tricuspid aortic valve with a reduction in cross-sectional area of about $80 \%$ ( Fig. 1). To generate the computational mesh, the volume of the stenosed aorta lumen which is described by a set of points on the external surface was reconstructed by taking cross-sections perpendicular to the centreline and using cubic splines to define the contours of each crosssection of the lumen. The full geometry representation was 
then completed by a sweeping operation of the reconstructed cross-sections along defined path curves. The conforming hexahedral curve-sided element mesh skeleton was generated using the Trelis mesh generation toolkit (CSIMSOFT, 2018. http://www.csimsoft.com/trelis.jsp). The domain was divided into several blocks in order to allow for different mesh refinements depending on the distance from the stenosis. The final grid used consists of 92,208 conforming curve-sided spectral elements within which the solution is expressed in terms of order $\mathrm{N}=7$ polynomials resulting in a total of about 47 million discretisation points.
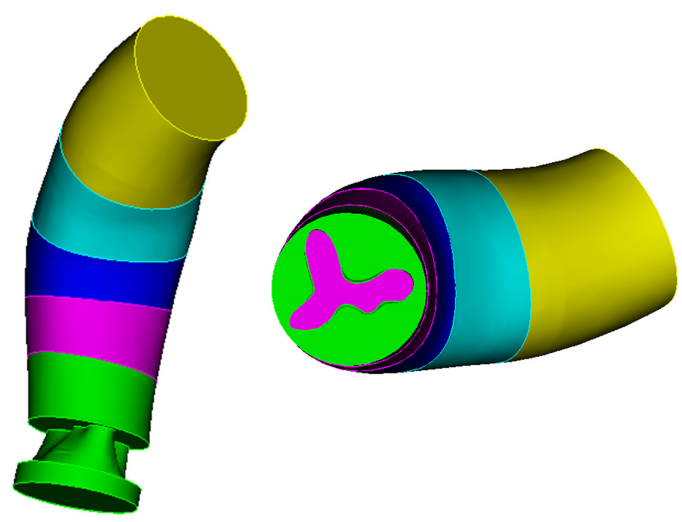

Fig. 1. Geometry of the ascending aorta divided into different blocks to define different mesh cell sizes. The orifice geometry represents a severly stenosed tricuspid valve.

The evolution of the transitional flow is governed by the incompressible continuity and momentum equations:

$$
\nabla \bullet \mathbf{v}=0
$$

$$
\frac{\partial \mathbf{v}}{\partial t}+\mathbf{v} \bullet \nabla \mathbf{v}=-\nabla p+\frac{1}{R e} \nabla \bullet \nabla \mathbf{v}
$$

with $\mathrm{t}, \mathbf{v}, p$, the nondimensional time, velocity and pressure and the Reynolds number $R e=\frac{U D}{\nu}=2100$ with $U=$ $0.407 \mathrm{~m} / \mathrm{s}$, the mean inflow velocity, $D=25 \mathrm{~mm}$, the inlet diameter and $\nu=4.8510^{-6} \mathrm{~m}^{2} / \mathrm{s}$, the kinematic viscosity. The flow rate imposed during in vitro PTV experiments is then of $12 \mathrm{~L} / \mathrm{min}$. This flow rate corresponds to a typical systolic flow rate pumped by an adult heart. The Reynolds number calculated at the orifice $R e_{o}=\frac{v_{j e t} d_{j e t}}{\nu}=3800$ with the mean jet velocity $v_{j e t}=1.65 \mathrm{~m} / \mathrm{s}$ and the jet diameter at the orifice $d_{j e t}=11 \mathrm{~mm}$. The reference length and velocity for nondimensionalisation are the inlet radius and twice the mean inflow velocity, respectively. The simulations were carried out using the open source incompressible flow spectral element solver NEK5000 [21]. The solution is expressed in terms of $\mathrm{N}$-th order tensor-product Lagrange polynomials based on the Gauss-Lobatto-Legendre quadrature points. The third-order temporal integration is based on a semi-implicit formulation using Backward Differentiation Formulas (BDF), where the convective terms are treated explicitly and the viscous term is treated implicitly [11]. A time-varying Dirichlet boundary condition (BC) is imposed at the inflow, while the outflow
$\mathrm{BC}$ is similar to the one found in [29]. In order to ensure that the flow characteristics at the outflow boundary are always pointing outwards, the flow is forced to leave the domain by imparting a positive velocity divergence in the last elements attached to the boundary. Since the geometry of the domain used for DNS is the exact same geometry as the one provided for the manufacturing of the stenosed aorta model made of rigid silicone and used in the experiments with the exception that the domain is cut before the aortic arch and as the flow is not pulsatile and no backflow at the level of the outflow boundary is noticed during the experiments, this imposed outflow $\mathrm{BC}$ is realistic and in line with the experimental conditions. The non-trivial time-varying inflow velocity is obtained from the measured profiles of each velocity component from in vitro 3D-PTV experiments. Although the flow rate imposed by the pump during the measurements is constant, it was observed that the profiles were not perfectly steady in time. In addition, the shape of these profiles is not axisymmetric unlike the ones predicted by Poiseuille or Womersley solutions and commonly used as inflow velocity profiles for simulations [20], [29]. We have noted that this unsteady behaviour caused important differences in the flow development downstream of the stenosis. In particular, the instability of the jet issuing from the orifice varied significantly between a steady inflow boundary condition and the time varying one (see video XY in supplementary materials). For this reason, a proper orthogonal decomposition (POD) using the method of snapshots for each velocity component in the inflow plane was performed [5]. The first 100 snapshots of this decomposition were used for the reconstruction of the profiles. The number of retained modes was chosen such that the sum of the 100 eigenvalues represents $95 \%$ of the total value over the full set of eigenvalues. The reconstructed velocity profile is then expressed as:

$$
v_{i}^{i n}(x, y, t)=\sum_{j=1}^{100} a_{j}(t) M_{i, j}(x, y)
$$

where

$$
M_{i, j}(x, y)=v_{i_{m e a s}} V_{j} \frac{1}{\sqrt{\lambda_{j}}}
$$

is the jth mode for the ith velocity component and

$$
a_{j}(t)=\sqrt{\lambda_{j}} V_{j}
$$

is the time-dependent coefficient multiplying each mode, while $\lambda_{j}$ and $V_{j}$ are respectively the $\mathrm{j}^{\text {th }}$ eigenvalue and the $\mathrm{j}^{\text {th }}$ eigenvector of the autocorrelation matrix $\mathbf{R}$ defined as:

$$
\mathbf{R}=v_{i_{\text {meas }}}^{T} v_{i_{\text {meas }}} \in \mathbb{R}^{N_{\text {snap }}} \times \mathbb{R}^{N_{\text {snap }}} .
$$

The modes extracted from the POD are fitted to an analytical function so that they can be imposed as boundary condition in 


$$
\begin{aligned}
\hat{M}=p_{k} & \sum_{l} \sum_{\gamma} \exp \left(-r^{2}+2 r l(\sin \theta \sin \gamma+\cos \theta \cos \gamma)-2 l^{2}\right. \\
& +\exp \left(-r^{2}-2 r l(\sin \theta \sin \gamma+\cos \theta \cos \gamma)-2 l^{2}\right) \\
& +\exp \left(-r^{2}+2 r l(\sin \theta \sin \gamma-\cos \theta \cos \gamma)-2 l^{2}\right) \\
& +\exp \left(-r^{2}+2 r l(-\sin \theta \sin \gamma+\cos \theta \cos \gamma)-2 l^{2}\right) \\
& +\exp \left(-r^{2}\right) \\
& l \\
& \in\{0.25,0.5,0.75\} \text { and } \gamma \in\left\{0, \frac{\pi}{6}, \frac{\pi}{4}, \frac{\pi}{3}\right\}
\end{aligned}
$$

3 with $r$ the radius and $\theta$ the azimuthal angle of the inflow 4 cross-section. Through a fitting procedure, the coefficients $5 \quad p_{k}$ are determined such that the squared difference between 6 the value given by the RBF and the expected value of the considered mode is minimised. The streamwise velocity profile is constrained such that the integral of this component over the inflow cross-section is equal to the flow rate measured during the experiments. The flux for the other two velocity components is constrained to be null. The shape of the RBF that fits the reconstructed modes is shown at four time instants in Fig. 2 (c), (d), (e), (f).

The smallest resolved scale in our DNS is about 25 microns. The minimum Kolmogorov length scale $\eta$ along the centreline of the ascending aorta is equal to 75 microns and is located at a distance from the stenotic orifice of about half the radius at the sinotubular junction.

Within the scope of this study, we mimic the effect of limited spatial resolution for in vitro and in vivo flow measurements by spatially filtering the DNS data. Thereby, the DNS velocity fields is filtered using an isotropic Gaussian filter as the one commonly used for large-eddy simulation (see Equations $11,10,21$ ) [25]. The cut-off length $\Delta$ was chosen to match the resolution usually achieved in in vitro PTV or 4D fow MRI measurements, namely $1.5 \mathrm{~mm}, 1 \mathrm{~mm}$ and 750 $\mu \mathrm{m}$ (see Fig. 3 (b)).

\section{B. In silico $3 D-P T V$}

The velocity along trajectory points obtained from threedimensional particle tracking velocimetry with perfectly passive tracer particles is extracted from the DNS velocity field. The seeding density of tracer particles is chosen such that three mean inter-particle distances $\delta$ match the previously mentioned cut-off lengths $\Delta$ (cf. Fig. 3 (c)). Thus, a seeding density of about 250, 1000 and 2300 particles per cubic centimetre results in a mean inter-particle distance of respectively $1.5,1$ and 0.75 millimetre (see video in supplementary material). In order to calculate the relevant quantities for analysis from the trajectory-based velocity, an interpolation of the velocity field is performed using a Gaussian convolution with a window width equal to $1.7 \delta$ [1]. In this work, the centreline of the aorta geometry is the location where the three-dimensional flow information is taken (cf. Fig. 3 (e)). In fact, pressure catheter and Doppler measurements are usually made along this centreline for the assessment of stenosis severity or for the characterisation of the artificial valve performance. The centreline can be determined as the weighted shortest path between the two extremities of the ascending part of the aorta. It is computed by minimising the line integral of the inverse of the maximal inscribed sphere radius calculated at each vertex of the Voronoï diagram generated from the points on the surface boundary of the ascending aorta [24].

\section{In vitro $3 D-P T V$}

In vitro three-dimensional particle tracking velocimetry experiments are carried out in two anatomically accurate silicone replicas of two stenosed aorta geometries. Details on the apparatus and material used to make the measurements can be found in [9] and [14]. The fluid used in both cases is a mixture of water ( $48 \%$ by weight), glycerine (37\% by weight) and sodium chloride ( $15 \%$ by weight) that behaves as a Newtonian fluid with kinematic viscosity $\nu=4.8510^{-6} \mathrm{~m}^{2} / \mathrm{s}$ and density $\rho=1200 \mathrm{~kg} / \mathrm{m}^{3}$ and a refractive index of 1.41 . Images are recorded with a CMOS camera at a frame rate of $2 \mathrm{kHz}$ and the time interval between two instantaneous velocity fields after the interpolation based on Gaussian convolution is around 3 ms. The geometry of the first rigid silicone model is the one shown in Fig. 3 (f) and the one used in the direct numerical simulation. The same nondimensionalisation of the fields of interest is performed and a Gaussian convolution similar to that applied to the in silico trajectory point velocity is carried out. As mentioned above, the velocities measured upstream of the stenosis are used to impose the inflow boundary condition for DNS. To test the applicability to a wider range of cases of the new models proposed for subvoxel stress and pressure gradient estimation, in vitro measurements under pulsatile conditions in the second aorta replica representing the same aorta geometry made of flexible silicone are performed. The stenosis geometry for this case is concentric and the reduction in cross-section area is equivalent to that of the geometry presented in Fig. 1. In vitro pressure measurements are also performed at various locations using a sensor on a catheter. For further details on the experimental setup and conditions, the reader is referred to [14]. The interpolation method used to express the velocity onto a Cartesian grid is similar to that of the previous in silico and in vitro PTV case except that the time-averaging operation is replaced by a phase-averaging one.

\section{THEORY}

The theoretical part of this paper starts with a rigorous derivation of a differential equation for pressure gradient evaluation based on the Navier-Stokes equation and a description of the filtering and ensemble-averaging operations applied to it. Then, closure models for the subvoxel-scale stress term are introduced. The final one-dimensional form of the pressure equation is presented and the hypotheses made to formulate the models are described.

\section{A. Differential Equation for Pressure Gradient Evaluation}

Since flow measurements resolved down to the Kolmogorov length scale remain unavailable and since in clinical practice, ensemble-averaged quantities are considered, we will assume 

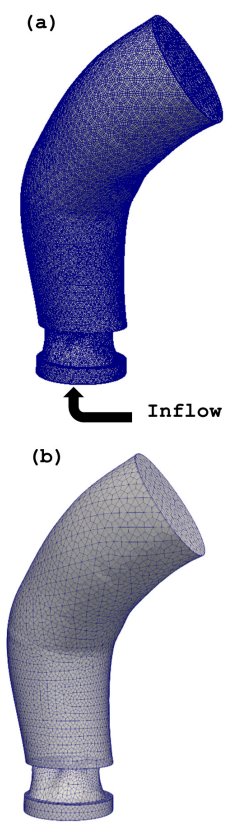
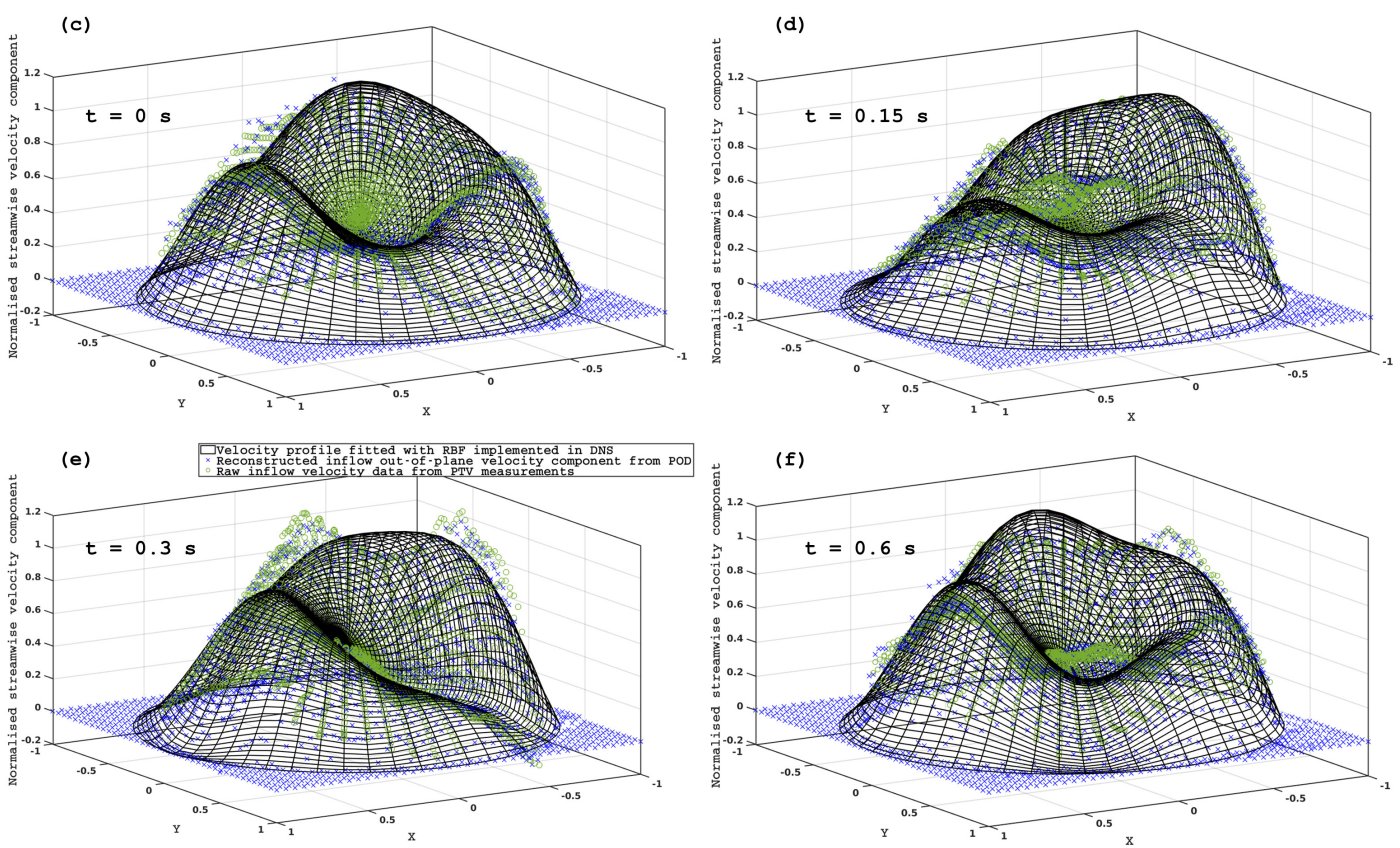

Fig. 2. (a) Ascending aorta geometry discretised with $47.210^{6}$ points. (b) Spectral element skeleton consisting of 92,208 conforming hexahedral elements. Fitted inflow velocity profile retrieved from proper orthogonal decomposition (POD) of the velocity data obtained from in vitro PTV measurements at (c) $\mathrm{t}=$ $0 \mathrm{~s}$, (d) $\mathrm{t}=0.15 \mathrm{~s}$, (e) $\mathrm{t}=0.3$, (f) $\mathrm{t}=0.6 \mathrm{~s}$.

that the behaviour of the flow quantities obtained with these under-resolved flow measuring techniques is described by the ensemble-averaged and spatially filtered Navier-Stokes vector equation expressed as follows:

$$
\begin{gathered}
\underbrace{\overline{\nabla \tilde{p}}}_{\text {Pressure gradient }}=\underbrace{-\overline{\mathbf{v} \bullet \nabla \tilde{\mathbf{v}}} \underbrace{+\frac{1}{R e} \overline{\nabla e} \bullet \tilde{\mathbf{v}}}_{\text {Viscous term }}}_{\text {Advective term }} \\
\underbrace{-\overline{\nabla \bullet \tilde{\tau}^{\mathbf{r}}}}_{\text {Subvoxel-scale stress }} \\
\underbrace{-\frac{\overline{\partial \tilde{\mathbf{v}}}}{\partial t}}_{\text {Acceleration term }}
\end{gathered}
$$

where tilde refers to a filtered quantity:

$$
\begin{aligned}
\tilde{\phi}(\mathbf{x}, t) & =\int_{\Omega} \phi(\mathbf{y}, t) G(|\mathbf{x}-\mathbf{y}|) d \mathbf{y} \\
& =\sum_{k \in \mathbb{Z}^{3}} \hat{\phi}(\mathbf{k}, t) \hat{G}(|\mathbf{k}|) \exp (i \mathbf{k x}) .
\end{aligned}
$$

The filter kernel $G$ is Gaussian and defined in physical and spectral spaces in a similar way to the one used for largeeddy simulations [25]:

$$
\begin{gathered}
G(|\mathbf{x}-\mathbf{y}|)=\frac{\sqrt{12}}{\sqrt{2 \pi} \Delta} \exp \left(\frac{-6|\mathbf{x}-\mathbf{y}|^{2}}{\Delta^{2}}\right), \\
\hat{G}(|\mathbf{k}|)=\exp \left(\frac{-|\mathbf{k}|^{2} \Delta^{2}}{24}\right),
\end{gathered}
$$

with $\Delta$, the cut-off length or voxel size.

Ensemble-averaging is performed in the following way:

$$
\bar{\phi}=\frac{1}{N_{t}} \sum_{k=1}^{N_{t}} \phi_{\text {inst }}(x, t+k \Delta t)
$$

with $N_{t}$, the number of time instances and $\Delta t$ the time interval between those instances. In the case of constant flow rate, $N_{t}$ is equal to 450 and $\Delta t=3 \mathrm{~ms}$. Under pulsating inflow conditions, $N_{t}$ is the number of cycles considered which is 60 and $\Delta t=1 \mathrm{~s}$ is the period of the pulse.

By virtue of Reynolds decomposition, the advective term of Eq. 8 is decomposed and the Reynolds stress term appears. The ensemble-averaging operation is a Reynolds operator which is commutative with time and space-derivative.

$$
\begin{aligned}
\overline{\nabla \tilde{p}} & =\left[-\overline{\tilde{\mathbf{v}}} \bullet \nabla \overline{\tilde{\mathbf{v}}}+\frac{1}{R e} \nabla \bullet \nabla \overline{\tilde{\mathbf{v}}}-\nabla \bullet \tilde{\mathbf{R}}\right] \\
& -\left[\nabla \bullet \tilde{\tau}^{\mathbf{r}}\right]-\frac{\partial \overline{\tilde{\mathbf{v}}}}{\partial t},
\end{aligned}
$$

with $\tilde{\mathbf{R}}$ the filtered Reynolds stress tensor:

$$
\tilde{R}_{i j}=\overline{\tilde{v}_{i}^{\prime} \tilde{v}_{j}^{\prime}}=\overline{\tilde{v}_{i} \tilde{v}_{j}}-\overline{\tilde{v}_{i}} \overline{\tilde{v}_{j}},
$$

and $\tilde{\mathbf{v}}^{\prime}$ the filtered fluctuating velocity.

The penultimate term on the right-hand side of Eq. 13 represents the divergence of the so-called tensor of subvoxel-scale (SVS) stress. This SVS stress is expressed in the following way:

$$
\overline{\tilde{\tau}_{i j}^{r}}=\overline{\overline{v_{i j} v_{i j}}-\tilde{v}_{i j} \tilde{v}_{i j}} \text {. }
$$

\section{B. Model Derivation}

Since the direct and rigorous computation of SVS stress is only possible by filtering fully resolved DNS velocity fields, a closure model based on resolved flow quantities is needed to 


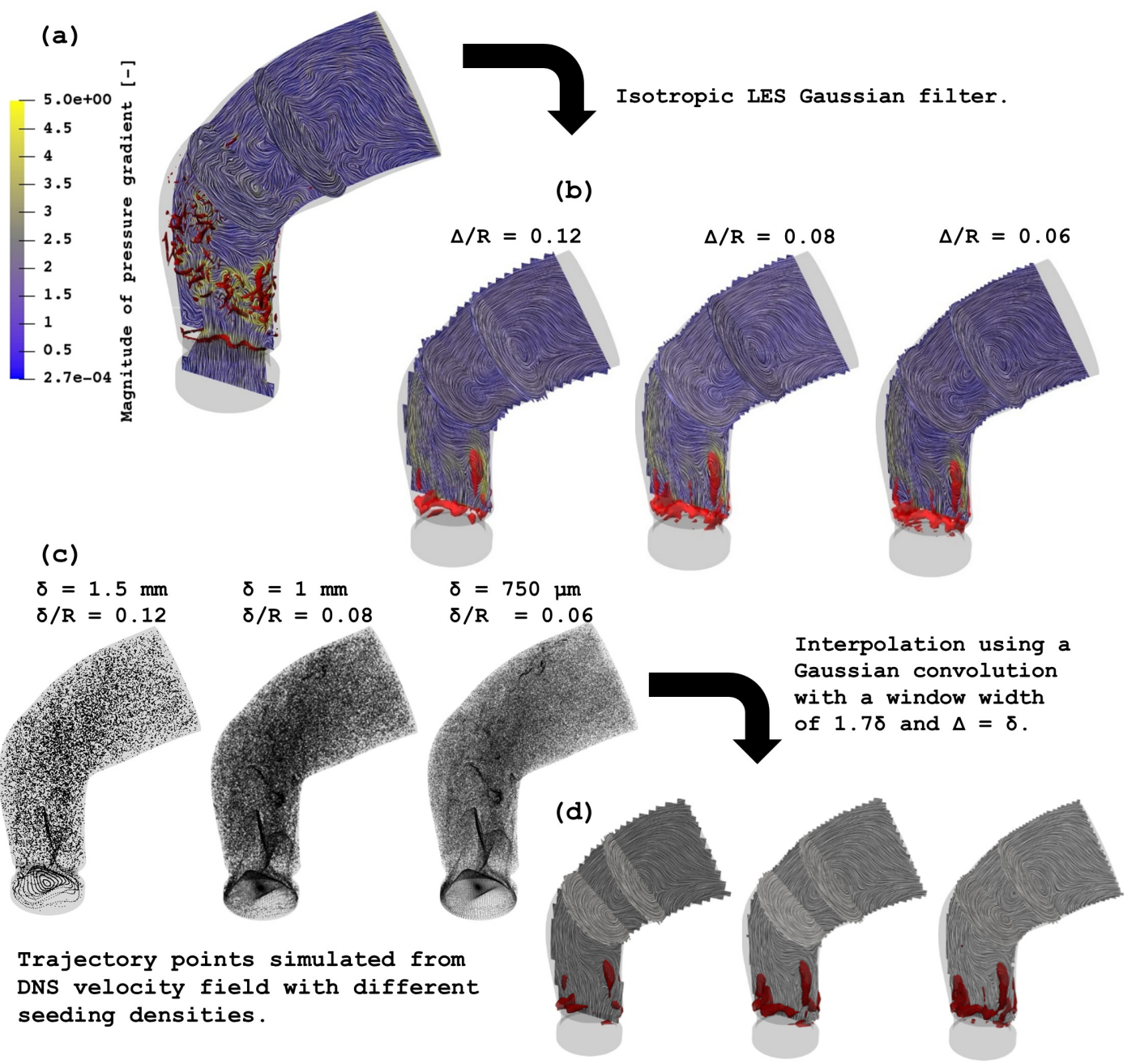

(f)

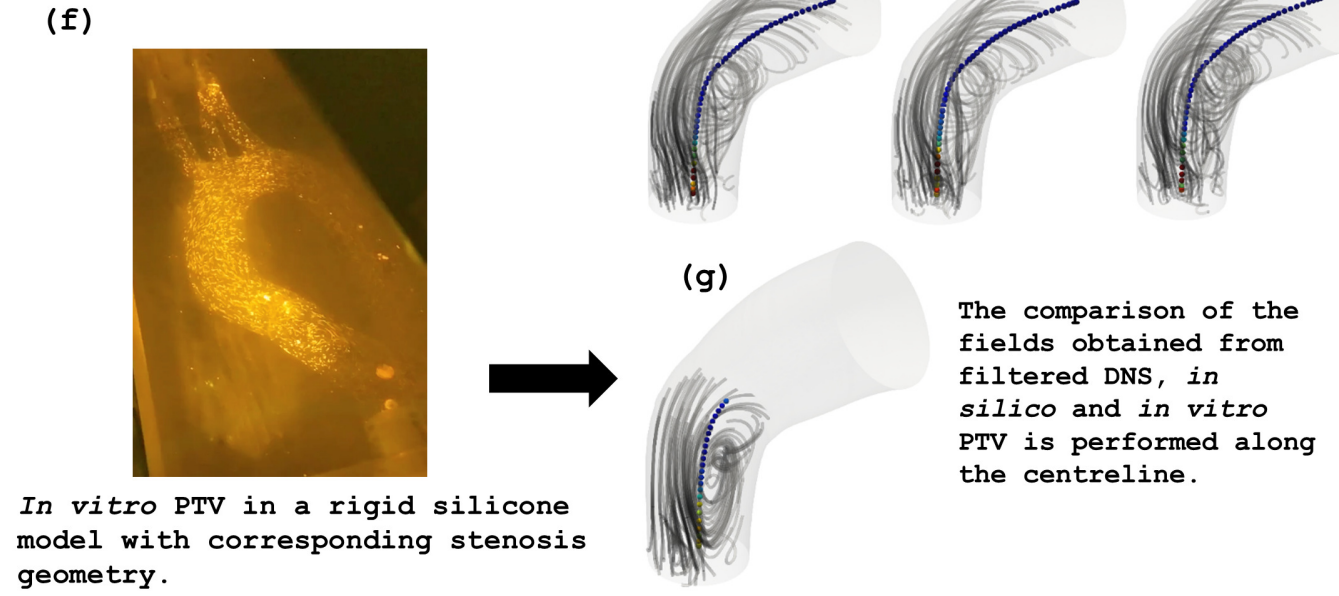

Fig. 3. Illustration of the three methods of investigation (a) DNS of the flow downstream of a severe stenosis geometry (method 1). Linear integral convolution visualisation of instantaneous velocity field coloured with the magnitude of pressure gradient in three planes shows flow patterns complemented with isosurfaces of positive Q values highlighting coherent vortical structures. (b) Time-averaged flow features from DNS fields filter at three cut-off lengths $(\Delta=$ $1.5 \mathrm{~mm}, 1 \mathrm{~mm}$ and $750 \mu \mathrm{m}$ ) brought out by planes with linear integral convolution of velocity field and iso-surfaces of positive Q values. (c) Passive tracers advected using DNS velocity field (method 2). Three seeding densities were used such that the mean inter-particle distance $\delta$ is equal to the filter cut-off lengths of the filtered DNS. (d) Flow features as in (b) obtained from interpolation of velocity on the simulated trajectory points. The size of the voxel used for the interpolation is equal to $\delta$. (e) Streamlines generated from the interpolated and time-averaged in silico velocity field and reconstructed centreline of the ascending part of the considered aorta geometry. (f) Illuminated tracer particles during in vitro experiments in the rigid silicone model of the stenosed aorta (method 3). (g) Streamlines generated from the interpolated in vitro velocity field in the first half of the ascending aorta and centreline. 
with $\tilde{\Delta}=\Delta / R$, the non-dimensional voxel size and $\tilde{\mathbf{S}}_{\text {inst }}$, the filtered rate-of-strain tensor:

$$
\tilde{\mathbf{S}}_{\mathrm{inst}}=\frac{1}{2}\left(\nabla \tilde{\mathbf{v}}+\nabla \tilde{\mathbf{v}}^{T}\right) .
$$

$$
\begin{aligned}
\overline{\nabla \bullet \tilde{\tau}^{\mathbf{r}}} & =\left(C_{l, 1} \tilde{\Delta}\right)^{2} \overline{\nabla \bullet \tilde{\mathbf{S}}_{\text {inst }} \tilde{\mathbf{S}}_{\text {inst }}}+C_{l, 2} \tilde{\Delta}^{2} \bar{\nabla} \tilde{\mathbf{\Omega}}_{\text {inst }} \tilde{\mathbf{\Omega}}_{\text {inst }} \\
& +C_{l, 3} \tilde{\Delta}^{2} \nabla \bullet\left(\frac{\tilde{\mathbf{S}}_{\text {inst }} \tilde{\mathbf{S}}_{\text {inst }} \tilde{\Omega}_{\text {inst }} \tilde{\Omega}_{\text {inst }}}{\left\|\tilde{\mathbf{S}}_{\text {inst }}\right\|^{2}}\right) \\
& +C_{\varepsilon} \operatorname{Re} \tilde{\Delta}^{2} \nabla \bullet\left(\overline{\left.\tilde{\mathbf{S}}_{\text {inst }} \odot \tilde{\mathbf{R}}\right),}\right.
\end{aligned}
$$

26 with $\tilde{\Omega}_{\text {inst }}$, the resolved rotation-rate tensor:

$$
\tilde{\Omega}_{\text {inst }}=\frac{1}{2}\left(\nabla \tilde{\mathbf{v}}-\nabla \tilde{\mathbf{v}}^{T}\right),
$$

and Equations 19 and 20 stem from the decomposition of the product of the velocity gradient tensor with its transpose:

$$
\begin{aligned}
& \overline{\nabla \tilde{\mathbf{v}} \nabla \tilde{\mathbf{v}}^{T}}=\overline{\tilde{\mathbf{S}}_{\text {inst }} \tilde{\mathbf{S}}_{\text {inst }}}+\tilde{\boldsymbol{\Omega}}_{\text {inst }} \tilde{\Omega}_{\text {inst }} \\
& +\frac{\overline{\tilde{\mathbf{S}}_{\text {inst }} \tilde{\mathbf{S}}_{\text {inst }} \tilde{\boldsymbol{\Omega}}_{\text {inst }} \tilde{\boldsymbol{\Omega}}_{\text {inst }}}}{\left\|\tilde{\mathbf{S}}_{\text {inst }}\right\|^{2}} .
\end{aligned}
$$

In addition, in line with the objective of this study, the pressure gradient vector needs to be projected along the aorta centreline. The differential equation for pressure gradient evaluation can be written in its final one-dimensional form:

$$
\begin{aligned}
\overline{\nabla \tilde{p}} \bullet \mathbf{t} & =\left[\left(-\overline{\tilde{\mathbf{v}}} \bullet \nabla \overline{\tilde{\mathbf{v}}}+\frac{1}{R e} \nabla \bullet \nabla \overline{\tilde{\mathbf{v}}}\right)-\nabla \bullet \tilde{\mathbf{R}}\right] \bullet \mathbf{t} \\
& -\left[\overline{\nabla \bullet \tilde{\tau}^{\mathbf{r}}}\right] \bullet \mathbf{t}-\frac{\partial \overline{\tilde{\mathbf{v}}}}{\partial t} \bullet \mathbf{t} .
\end{aligned}
$$

with $\mathbf{t}$ the tangent vector to the centreline. The last term on the right-hand side of Eq. 23 is equal to zero in the case of constant imposed flow rate.

The approach followed in this study consists of optimising the parameters of Equations 23, 16, 17, 19, 20 for different voxel sizes by calculating the various terms in the right-hand side of these equations from in silico PTV velocity fields and projecting them onto the tangent vector of the centreline. Pressure gradient fields from filtered DNS as well as subfilterscale stress retrieved from DNS data are used as reference values. Thus, the left-hand side terms of Equations 16, 19, 23 are obtained from filtered DNS fields whereas the terms on the right-hand side of these equations are all evaluated from in silico or in vitro PTV. The parameters $C_{i}$ appearing in Equations 16, 17 19, 20 are obtained by a linear least-square optimisation using an interior-point method [2] and performed over a set of around 4500 points located in a tube centred on the centreline and under constraints on the integral of the various quantities along the centreline. This problem can be formulated in the following way:

$$
\begin{aligned}
\min _{C_{i}} f\left(C_{i}\right) & =\min _{C_{i}}\left\|M C_{i}-d\right\|_{2}^{2} \\
& =\min _{C_{i}} \frac{1}{2} C_{i}^{T} H C_{i}+r^{T} C_{i}
\end{aligned}
$$

such that $A_{e q} C_{i}=b_{e q}$.

with $f\left(C_{i}\right)$, the objective function; $M$, the matrix with the terms on the right-hand side of Equations 16, 17, 19, 20, 23; $d$, the vector with the reference value of pressure gradient and subvoxel-scale stress obtained from DNS; $\mathrm{H}$, the Hessian matrix $2 M^{T} M ; r=-2 M^{T} d ; A_{e q}$, the matrix with the values of the integral along the centreline of the different terms; $b_{e q}$, the reference pressure difference and the integral over the centreline of the reference SVS stress. To measure how close the computed minimum is to the optimal value as part of the interior-point algorithm, the first-order optimality is tracked. A relative tolerance of $10^{-14}$ for this measure is set as a stopping criterion for the iterations. The first-order optimality measure when constraints are present is based on the Karush-KuhnTucker conditions, which can be expressed as:

$$
\left\|\nabla_{C_{i}} L\left(C_{i}, \lambda\right)\right\|_{\infty}=\left\|\nabla f\left(C_{i}\right)+A_{e q}^{T} \lambda\right\|_{\infty}
$$


with $\lambda$, the Lagrange multiplier vector from the equality constraint on the integral values.

In order to compare the performance of the two proposed models for the three considered resolutions, the coefficient of determination is calculated. As defined in Eq 26, this coefficient represents the percentage of the variance of the observed data (i.e. the pressure gradient and the SVS stress) explained by the model. The computation of such a coefficient is performed based on a set of about 4500 points distributed in a tube centred on the centreline of the aorta and with a radius of approximately 0.8 times the aorta radius. The reason for choosing this volume is to have a sufficient number of data points for a robust optimisation of parameters general enough to estimate SVS stress and pressure gradient in the bulk of the flow. Indeed, a convergence study on the $R^{2}$ values was performed to make sure that the number of sampling points for the optimisation is sufficient. We will show in the results section below the estimated pressure gradient and SVS stress along the centreline, which is a subset of the whole set of points considered for optimisation.

$$
R^{2}=1-\frac{\sum_{i}\left(\phi_{i}-\hat{B}_{i}\right)^{2}}{\sum_{i}\left(\phi_{i}-\overline{\phi_{i}}\right)^{2}},
$$

$\hat{B}_{i}$ is the expected regression line with slope equal to $1, \phi_{i}$ are the values of pressure gradient or SVS stress evaluated with the models at the sampling points and $\overline{\phi_{i}}$ is the of average value of the reference pressure gradient or SVS stress.

Furthermore, in order to assess the sensitivity of the two models at the three resolutions to a variation in the parameter value or error in the evaluated terms, we study the variation of the coefficient of determination as a function of an imposed error ranging from $-40 \%$ to $+40 \%$ which is a conservative choice because it is larger than typical experimental errors in velocity data and related terms of Eq. 23 ([9], [14], [19]). Since the models for pressure gradient estimation and the new model for SVS stress evaluation use more than one optimised parameter, a combined $R^{2}$ is calculated by computing the convolution product of the 6th degree polynomials fitted to the $R^{2}$ distribution depending on the imposed error for each term of the models (cf. Equations 23, 16, 17, 19, 20). Finally, our approach is based upon the assumption that the velocity field obtained from the Gaussian filtering operation applied to the DNS velocity is consistent with the velocity field interpolated on a structured Cartesian grid drawn from the Gaussian convolution applied to the trajectory point velocities.

\section{RESULTS AND DisCUSSION}

This section presents the results of the model constant optimisation and compares the estimated pressure and SVS stress with the reference DNS-based values to draw conclusions on the validity of models in relation to the three considered voxel sizes. It also provides a physical interpretation for the new model and discusses the applicability of these models to in vitro PTV measurements. It then compares pressure drop estimates obtained with other pressure models from the literature that are used in clinical practice. Finally, the validity of the new approach is tested for more realistic conditions and the relevance for clinical application of such a novel approach is highlighted by comparing the discrepancies from the direct pressure measurement between the proposed models and the method proposed by Ha et al. ([15], [16]).

\section{A. Model Optimisation, Performance and Physical Interpre- tation}

Tables I and II provide the values of the optimised coefficients.

The performance of the modelled projected pressure gradient and subvoxel-scale (SVS) stresses as compared to the reference values is assessed by considering the coefficient of determination $R^{2}$ given in Tables I and II calculated based on a sample of 4500 points. The optimisation of the above parameters is performed for the Equations 17, 20, 23 allowing for the evaluation of pressure gradient but also for the Equations 16, 19 representing the norm of the SVS stress tensor. The usefulness of the SVS stress models comes from the blood damage models, which are employed to quantify the detrimental effect blood flow exerts on the blood cells along their trajectory [9].

As far as the Smagorinsky-based models are concerned, we note in the first row of Tables I and II that the prediction quality of both the pressure gradient and the SVS stress reconstructed from in silico PTV is independent of the voxel size. The coefficient of determination $R^{2}$ representing the proportion (as a percentage of the variance of the reference data) of the in silico PTV estimate that agrees with the reference data is around $85 \%$ for the SVS stress estimate and $60 \%$ for the projected pressure gradient estimation. This trend suggests that irrespective of the voxel size and for optimised constants $C_{\nabla \bullet \tau, S m a g}$ and $C_{\tau, \text { Smag }}$ that do not strongly depend on the voxel size, these models would provide a good estimation especially for the SVS stress.

It is worth noting that the lowest resolution considered in this study corresponds to the one that can commonly be achieved with current in vitro and in vivo flow measurement techniques such as 3D-PTV or 4D-flow MRI [14], [15], [19].

With regard to the new model for the evaluation of pressure gradient from in silico PTV, the prediction quality is also independent of the the decreasing voxel size as evidenced by the values of $R^{2}$ in Table I. If we compare the performance of this new model with the Smagorinsky-based one, we notice that the coefficient of determination is $25 \%$ larger. Overall, the improvement in terms of fitting quality made with the new model is substantial.

The prediction quality for in silico SVS stress using the new model proposed in this study is reported in the first row of Table II. The coefficient of determination is $14 \%$ smaller at the two higher resolutions than the one at the voxel size of 0.12 . This suggests that this new model performs better when the resolution is coarser.

The physical interpretation of the new model for the evaluation of pressure gradient originates from the fact that in turbulent shear flows, many of the important kinematic and dynamical properties can be understood by describing the 
TABLE I

PARAMETERS OPTIMISED FOR THE TWO MODELS CONSIDERED FOR THE EVALUATION OF PRESSURE GRADIENT PROJECTED ONTO THE CENTRELINE.

\begin{tabular}{|c|c|c|c|c|c|c|c|c|c|}
\hline$\nabla p \bullet \mathrm{t}$ & $\overline{\Delta \bar{\Delta}}$ & 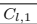 & $\begin{array}{ll}C_{l, 2} \\
\end{array}$ & $\overline{c C_{l, 3}}$ & $\overline{C_{\varepsilon}}$ & 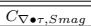 & 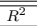 & $=\left\|\nabla_{C_{i}} L\left(C_{i}, \lambda\right)\right\| \times 10^{-14}$ & 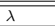 \\
\hline Smagorinsky & $\begin{array}{l}0.12 \\
0.08 \\
0.06\end{array}$ & $\mathrm{n} / \mathrm{a}$ & $\mathrm{n} / \mathrm{a}$ & $\mathrm{n} / \mathrm{a}$ & $\mathrm{n} / \mathrm{a}$ & $\begin{array}{l}13.25 \\
12.03 \\
8.74\end{array}$ & $\begin{array}{l}0.64 \\
0.61 \\
0.65\end{array}$ & $\mathrm{n} / \mathrm{a}$ & $\mathrm{n} / \mathrm{a}$ \\
\hline New model & $\begin{array}{l}0.12 \\
0.08 \\
0.06\end{array}$ & $\begin{array}{l}1.26 \\
1.96 \\
2.07\end{array}$ & $\begin{array}{l}-0.63 \\
2.14 \\
0.5\end{array}$ & $\begin{array}{l}0.028 \\
0.095 \\
-0.0064\end{array}$ & $\begin{array}{l}0.013 \\
0.094 \\
0.196\end{array}$ & $\mathrm{n} / \mathrm{a}$ & $\begin{array}{l}0.77 \\
0.75 \\
0.78\end{array}$ & $\begin{array}{l}0.96 \\
2.45 \\
0.116\end{array}$ & $\begin{array}{l}-0.014 \\
-0.305 \\
0.021\end{array}$ \\
\hline
\end{tabular}

TABLE II

OPTIMISED PARAMETERS FOR THE MODELS ALLOWING TO CALCULATE THE MAGNITUDE OF SUBVOXEL-SCALE STRESS ALONG THE CENTRELINE FROM 3D-PTV.

\begin{tabular}{|c|c|c|c|c|c|c|c|c|c|}
\hline 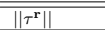 & $\overline{\Delta \bar{\Delta}}$ & 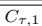 & $\overline{C C_{\tau, 2}}$ & 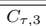 & $\overline{C_{\tau, 4}}$ & 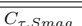 & 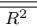 & $\overline{\|}\left\|\nabla_{C_{i}} L\left(C_{i}, \lambda\right)\right\| \times 10^{-14}$ & $\overline{\bar{\lambda} \lambda}$ \\
\hline \multirow[b]{2}{*}{ Smagorinsky } & 0.12 & & & & & 21.71 & 0.87 & & \\
\hline & 0.08 & $n / a$ & $\mathrm{n} / \mathrm{a}$ & $\mathrm{n} / \mathrm{a}$ & $n / a$ & 21.66 & 0.84 & $\mathrm{n} / \mathrm{a}$ & $\mathrm{n} / \mathrm{a}$ \\
\hline \multirow{3}{*}{ New model } & $\frac{0.06}{0.12}$ & 000 & & -3.2 & -0.018 & & 0.82 & & 0205 \\
\hline & 0.08 & 0.00 & 4.86 & 1.13 & 0.03 & \multirow{2}{*}{$\mathrm{n} / \mathrm{a}$} & 0.76 & 586 & $\begin{array}{l}-0.68 \\
6.68\end{array}$ \\
\hline & 0.06 & 0.00 & 5.52 & -1.52 & 0.1 & & 0.77 & 2.84 & 6.62 \\
\hline
\end{tabular}

flows in terms of individual events, namely the combination of coherent eddies, convergence and streaming zones [18]. In that respect, qualitative and mathematical criteria based upon threshold values for the second invariant of the velocity deformation tensor and the pressure in the flow have been proposed in Hunt et al. [18]. This representation can be useful since in this study, ensemble-averaged and under-resolved features of a transitional-to-turbulent flow can be characterised. The ensemble-averaged description of the flow at a fixed voxel size is then described through a combination of events representative of the fundamental nature of the underlying turbulence. Following this train of thought, because the new model for pressure gradient evaluation links pressure gradient and velocity deformation tensor through an equation, we sense that the optimisation of the different parameters could lead to the definition of unequivocal criteria that would allow to highlight the interplay between individual patterns in the type of shear-induced turbulent flows encountered in the aorta. Thereby, each term of Equations 13 and 20 can be interpreted in the following manner:

- $-\overline{\tilde{\mathbf{v}}} \bullet \nabla \overline{\tilde{\mathbf{v}}}$ represents the contribution to the pressure gradient in the flow due to streaming zones. As stated by Hunt et al. [18], in streaming zones, the flow is fast, not very curved and not diverging or converging strongly.

- $-\nabla \bullet \tilde{\mathbf{R}}$ represents the contribution to the pressure gradient due to momentum transport from Reynolds shear stresses.

- $-\tilde{\Delta}^{2} \nabla \bullet \overline{\tilde{\mathbf{S}}_{\text {inst }} \tilde{\mathbf{S}}_{\text {inst }}}$ represents the contribution to the pressure gradient due to irrotational straining motion in the flow also known as convergence zones $\left(T_{C_{l, 1}}\right)$. These zones are characterised by strong convergence and divergence of streamlines.

- $-\tilde{\Delta}^{2} \nabla \bullet \tilde{\boldsymbol{\Omega}}_{\text {inst }} \tilde{\Omega}_{\text {inst }}$ represents the contribution to the pressure gradient due to the action of coherent eddies in the flow $\left(T_{C_{l, 2}}\right)$ defined as strong swirling zones with vorticity.

- $-\operatorname{Re} \tilde{\Delta}^{2} \nabla \bullet\left(\overline{\tilde{\mathbf{S}}_{\text {inst }}} \odot \tilde{\mathbf{R}}\right)$ represents the contribution to the pressure gradient due to turbulent energy dissipation $\left(T_{C_{\varepsilon}}\right)$.
TABLE III

CONTRIBUTION OF EACH TERM OF THE EQUATION FOR PRESSURE GRADIENT ESTIMATION FOR THE TWO VARIANTS OF THE NEW MODEL. THE CONTRIBUTION IS CALCULATED FOR EACH VOXEL SIZE FROM THE RATIO BETWEEN THE INTEGRAL OF EACH TERM OVER THE CENTRELINE AND THE VALUE OF RECOVERED PRESSURE.

\begin{tabular}{clllllll}
\hline \hline$\frac{\int_{l} T_{i} \bullet \mathbf{\bullet} d l}{\Delta p_{\text {recovered }}}$ & $\tilde{\Delta}$ & $T_{\overline{\mathbf{v}} \bullet \nabla \overline{\mathbf{v}}}$ & $T_{\nabla \bullet \tilde{\mathbf{R}}}$ & $T_{C_{l, 1}}$ & $T_{C_{l, 2}}$ & $T_{C_{l, 3}}$ & $T_{C_{\varepsilon}}$ \\
\hline \multirow{2}{*}{ New model } & 0.12 & -2.46 & 1.61 & 1.93 & 0.35 & -0.01 & -0.43 \\
& 0.08 & -2.58 & 1.75 & 3.58 & -0.39 & -0.03 & -1.36 \\
& 0.06 & -2.6 & 1.92 & 3.77 & -0.1 & -0.002 & -2.09 \\
\hline \hline
\end{tabular}

Therefore, the new model weighs each of the last three terms above such that the combined action of the physical flow characteristics best matches the reference pressure gradient value. The contribution of each weighted term is presented in Table III. For this, each term is integrated over the centreline and divided by the value of recovered pressure in the ascending part of the considered stenosed aorta.

The term representing the influence of the turbulent straining motion zones on the pressure gradient prevails for the three voxel sizes and tends to counteract the action of the streaming advection (negative values) especially close to the stenosis. The absolute value of the relative contribution due to turbulent energy dissipation term $\left(T_{C_{\varepsilon}}\right)$ increases with the diminishing voxel size whereas the streaming zone term and the Reynolds stress term remain rather constant for the three voxel sizes.

\section{B. Sensitivity Analysis of The Models}

Figure. 4 presents the variation in the coefficient of determination for the two models and for the estimation of both the pressure gradient and SVS stress at the three resolutions. This analysis is useful to understand the impact of measurement errors or change in the parameter value on the predicted quantities of interest. The noticeable trend is the more important sensitivity of both models at the coarsest tested resolution whether for the pressure gradient or SVS stress estimates. In particular, as seen in Fig. 4 (a), the Smagorinsky-based model for the estimation of SVS stress magnitude is very sensitive to an over-estimation of its only term which is dependent on the calculation of the strain rate tensor magnitude. That being said, the new model for SVS stress estimation is much less sensitive even at the coarsest voxel size and for an error reaching $40 \%$ of the optimal state, the decrease in the $R^{2}$ is limited to a value of about $50 \%$. It is worth noting that unlike the Smagorinsky model at the larger voxel size, the new model for the evaluation of SVS stress at the intermediate and smaller voxel size is more robust to a general over-estimation than under-estimation of the various terms. With regard to the sensitivity of the two models for the pressure gradient estimation, the quality of prediction with the new model at the smaller voxel size is limited to a decrease in $R^{2}$ value of $20 \%$ when a $40 \%$ error is introduced. The Smagorinsky-based is more sensitive to errors or variation in its parameter value than the new model except at the lower resolution where the new model reaches negative $R^{2}$ values when a $30 \%$ over-estimation or under-estimation of its composing terms is introduced. Finally, in order to identify the term with the strongest influence on 


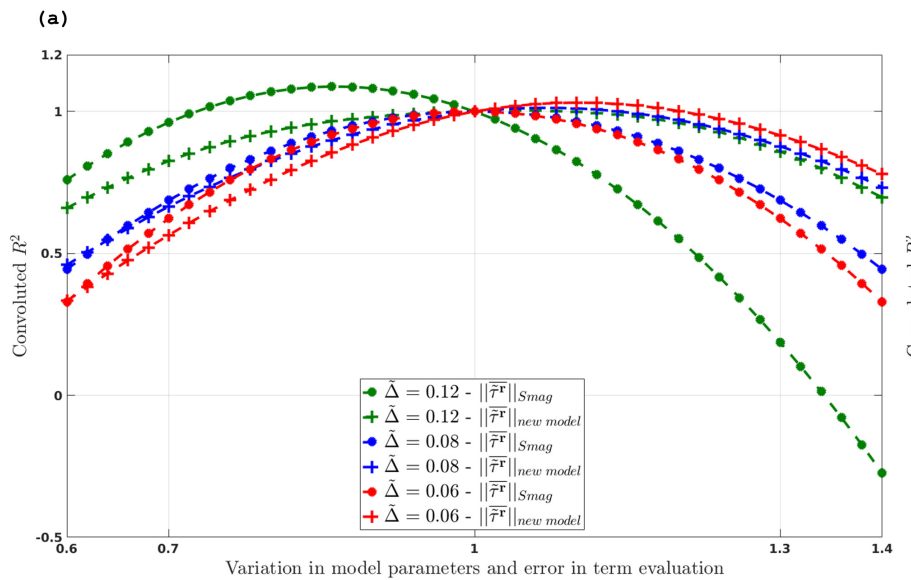

(b)

Fig. 4. (a) Graph of the convoluted $R^{2}$ as a function of the variation in the estimation of the different terms for the evaluation of SVS stress with both optimised models at the three resolutions. (b) Combined coefficient of determination for the two models estimating the projected pressure gradient for the three voxel sizes. The combined $R^{2}$ is derived from the convolution product of the variation of $R^{2}$ for each term of the two models

the quality of the prediction, the reader is referred to the supplementary figures. It can noted that for the SVS stress estimation from the new model, the second term of Eq. 19 has the highest impact on the convoluted $R^{2}$ curve. Regarding the pressure gradient evaluated from the Smagorinsky-based model, the advective term along with the term based on the calculation of the instantaneous magnitude of rate-of-strain tensor determine the bell shape of the convoluted $R^{2}$ curve except at the finest resolution where the advective term has the strongest influence on the $R^{2}$ values when variation in its value is imposed. As far as the pressure gradient estimate via the new model is concerned, the straining and streaming terms are the ones that most deflect the curve of the coefficient of determination from the ideal unitary value when errors in their evaluation is introduced.

\section{Comparison With The Corresponding In vitro Measure- ments}

As shown in Figs. 3 (f) and (g), the aforementioned models have been optimised and tested by calculating both pressure gradient and SVS stress from velocity fields measured with in vitro 3D-PTV. Owing to the seeding density of tracer particles that can be tracked in in vitro 3D-PTV measurements, the voxel size used for the Gaussian convolution corresponds to the largest voxel size employed for the validation of the models. The same nondimensionalisation as the one applied to the DNS and in vitro PTV has also been performed on velocity and tracer particle coordinates.

In Fig. 5 (a), we note that the value of the SVS stress estimated with the new model from in vitro PTV is in good agreement with the in silico reconstructed value and mostly with the value directly calculated from DNS. The estimate from the Smagorinsky-based model is matching the values obtained from in vitro PTV for the first half of the centreline. The observed discrepancies probably originate from calibration errors in the measurements and from the slightly different positioning of the centreline in the in vitro PTV data. It is important to note that the Smagorinsky-based model is more prone to error in the estimation given that it involves the third power of the instantaneous rate-of-strain tensor. In fact, the sensitivity analysis showed that the models for the SVS estimation at the coarsest voxel size give results that deviate more significantly from the reference SVS values when errors are introduced. Owing to the fact that the evaluation of the SVS stress magnitude with the Smagorinsky model is only based on the evaluation of a single term, the error in the instantaneous strain-rate to the power of three is crucial. In contrast, the estimate with the new model depends on the evaluation of three terms which tends to limit the combined error as evidenced by the better agreement of this model with the in silico PTV values. In [9], various models were used to assess the damage accumulated on blood cells. Those models integrate the value of stress along blood cell trajectory. For this reason, it is interesting to compare the value of the integral over the centreline of the SVS stress. We can note from Fig. 5 (b) that this value is overestimated by the Smagorinsky-based model due to the large errors introduced in the second half of the ascending aorta while a slight underestimation of the integrated SVS stress is noticed when using the new model from in vitro PTV measurements.

Figure 6 (a) compares the time-averaged pressure gradient along the centreline obtained from unfiltered and filtered DNS and the pressure gradient estimated from the models for in silico and in vitro PTV. It is noteworthy that the pressure gradient values evaluated from the two models are closer to the values calculated from the full DNS than from the filtered DNS. In fact, the lowest value at a non-dimensional distance of 0.5 from the first point of the centreline for the three models and the full DNS is about -0.5 . Yet, the underestimation of the pressure gradient close to the stenosis and the overestimation farther downstream might originate from a slightly different positioning of the centreline for the in vitro PTV measurements as compared to the reference positioning of the centreline for the in silico PTV data. In Fig. 6 (b), the value of the integral over the centreline of the pressure gradient estimated with the new model is in good agreement with reference pressure 
(a)

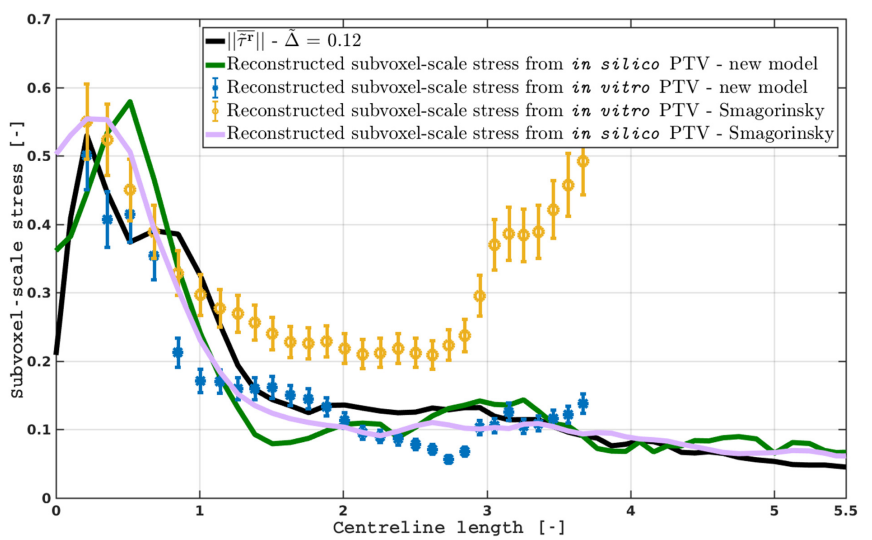

(b)

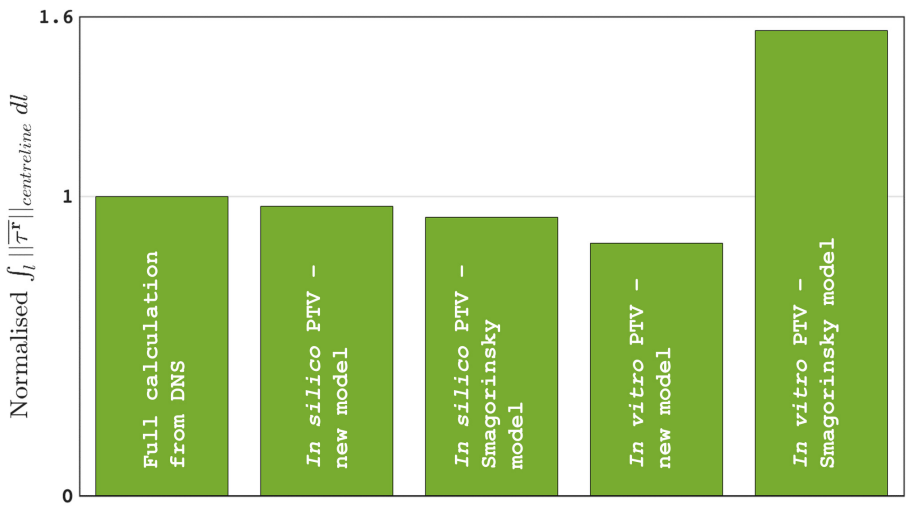

Fig. 5. (a) Comparison of time-averaged SVS stress along the centreline between direct calculation from DNS, from in vitro and in silico PTV estimates using two models (b) Normalised integral of the magnitude of time-averaged SVS along the centreline.

(a)

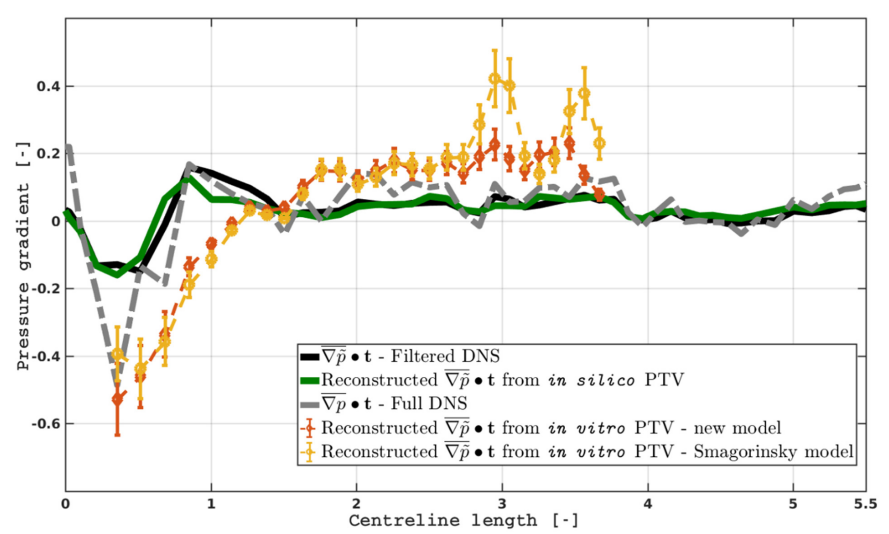

(b)

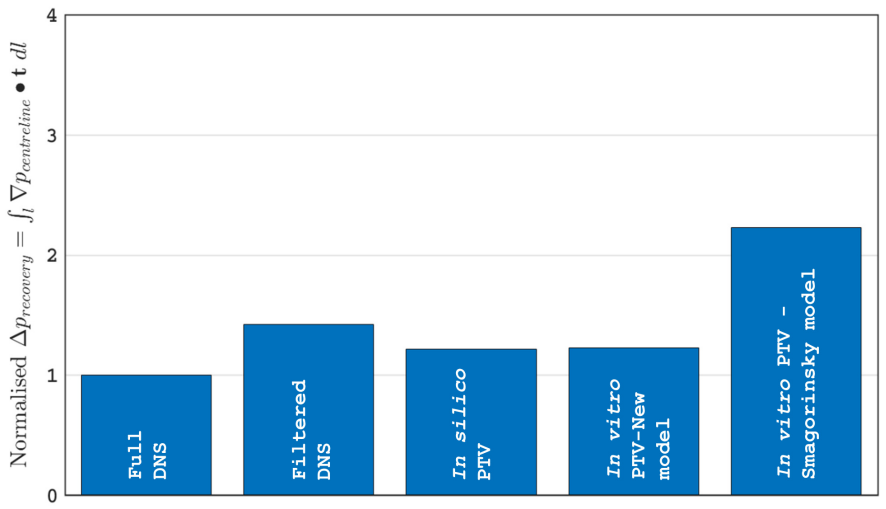

Fig. 6. (a) Comparison of projected pressure gradient along the centreline between the values obtained from full DNS, from filtered DNS, from in vitro and in silico PTV estimates using the two models (b) Time-averaged pressure difference in the ascending aorta and comparison of the irreversible pressure loss computed with DNS data to two methods used in clinical practice and one approach implemented for 4D flow MRI measurements.

difference calculated from the filtered DNS, in silico PTV and full DNS. However, the pressure difference calculated from the Smagorinsky-based model tends to be overestimated. In fact, as previously pointed out for the SVS stress estimation, this model is likely to introduce larger errors due to the third power of the instantaneous rate-of-strain tensor.

\section{Comparison With Other Pressure Drop Estimation Ap- proaches}

Figure 7 shows the performance of the three methods for the estimation of the irreversible pressure drop as compared to the time-averaged pressure difference between the inflow and the outflow cross-section computed from DNS and to the pressure difference obtained from the novel approach applied to in silico PTV data.

The latest model proposed by Hatoum et al. [17] offers the advantage of being independent of the stenosis effective orifice area and capturing the unsteadiness in pressure difference [17]. By applying this model to our steady case, we note a $75 \%$ underestimation with respect to the time-averaged pressure difference retrieved from DNS. The approach in Ha et al. [15],
[16] suggests that the irreversible pressure can be computed as the ratio between the integral of the production of turbulent kinetic energy over the whole volume and the flow rate. When applying this method to the DNS data in our case, the value obtained is about $35 \%$ smaller than the actual one.

Another tested model is the extended Bernoulli one. For this simple and easy-to-implement model, the irreversible pressure drop is evaluated by taking the square of the velocity at the vena contracta and multiplying it by a factor accounting for pressure recovery through the calculation of the ratio between the effective orifice area and the cross-sectional aorta area [15], [28]. The calculation of the pressure drop with this model results in a $40 \%$ underestimation, in line with the conclusions drawn for severe stenoses in other studies [22], [28]. It is then evident that the two models only based upon general flow features such as flow rate or velocity at one position do not allow to adequately estimate pressure difference.

The third method proposed by Ha et al. [15], [16] provides a value of irreversible pressure drop better than that given by the other two methods but it still underestimates the value by $30 \%$. Taking into account only the integral of the turbulence 
production term in the total energy balance and expressing this total energy between the upstream and downstream crosssections as the product of the flow rate and the pressure drop appear to be crude assumptions. Another shortcoming of this method as compared to the models proposed in this study regards the necessity of measuring quantity fields in the whole aorta volume whereas for the current approach, only data along the centreline are needed.

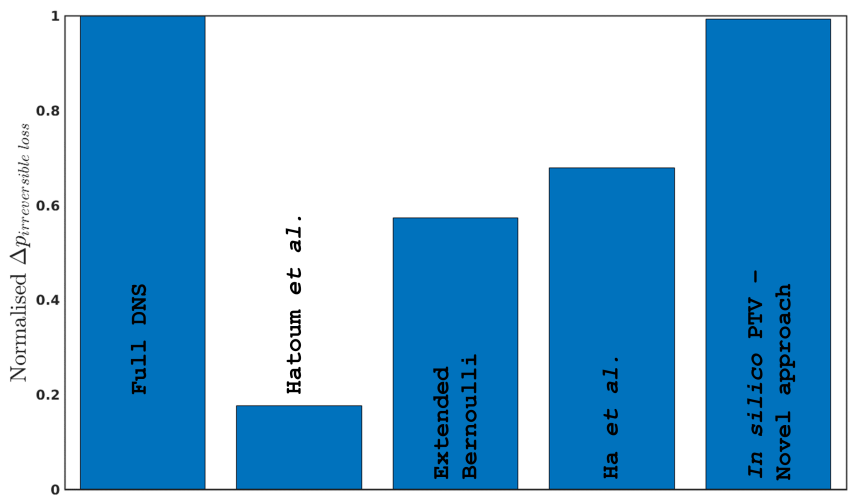

Fig. 7. Comparison of the time-averaged irreversible pressure loss computed from DNS to that calculated using the method of estimation proposed by Hatoum et al., to that obtained from the approach suggested by Ha et al. for the evaluation of irreversible pressure drop from 4D flow MRI measurements, to the value predicted the clinically applied indicator based on an extended Bernoulli law and to that based on the novel approach proposed in this study and applied to the in silico PTV data.

\section{E. Validation Based on a Pulsatile In vitro Case}

In order to show that under more realistic settings the proposed approach and optimised models still hold when it comes to evaluating the pressure gradient projected onto the tangent vector, a second set of in vitro 3D-PTV data is investigated (see section II C). The Reynolds number for this pulsatile case ranges from 20 to 1850 . In fact, with this second in vitro case, we investigate the flow downstream of a concentric severe stenosis model in a flexible silicone replica of aorta under pulsatile conditions. For these experiments, the pressure is measured using a sensor on a catheter at different locations along the centre axis in the ascending aorta. The phase-averaged pressure difference between the measured pressure at the two most extreme location points is the benchmark value used to verify the validity of the models for another case. The pressure gradient is evaluated from the in vitro 3D-PTV data using the differential equation and the two models. The integral over the centreline of the estimated pressure gradient gives the pressure difference at one time instant over the cycle.

Figure 8 compares the irreversible pressure drop in the model of ascending aorta measured with the pressure catheter to the value obtained by integrating the pressure gradient over the centreline using Eq. 23 and the two models at six instants during the first half of the pulse. The value of pressure difference retrieved at the same six instants using the approach suggested in Ha et al. [15], [16] is also presented in Fig 8. It can be seen that the two models provide a good estimate of the phase-averaged pressure difference, while the estimation based on the volume integral of turbulence production gives larger errors. In fact, the mean and the maximum error at the six considered time instants using the latter approach are respectively of 99 and $172 \mathrm{~Pa}$. The mean error thus represents $60 \%$ of the reference pressure difference measured with the pressure sensor and averaged over the pulse. The mean error using the new model is reduced to $23.7 \%$ of the pulse-averaged reference pressure while this error represents $38.5 \%$ of the pulse-averaged reference pressure in the case of the Smagorinsky-based approximation. It is noteworthy that the interpolation of the velocity data from the in vitro PTV experiments is performed on a Cartesian grid at the coarsest voxel size of $1.5 \mathrm{~mm}$. We showed that the two proposed models are more sensitive to errors in the evaluation of the different terms at this coarser voxel size, especially the Smagorinsky-based due to the presence of the third power of the instantaneous strain rate tensor. Despite this sensitivity to measurement errors of the two models at the larger voxel size, the error reduction compared to the prediction based on the approach by Ha et al. is still significant.

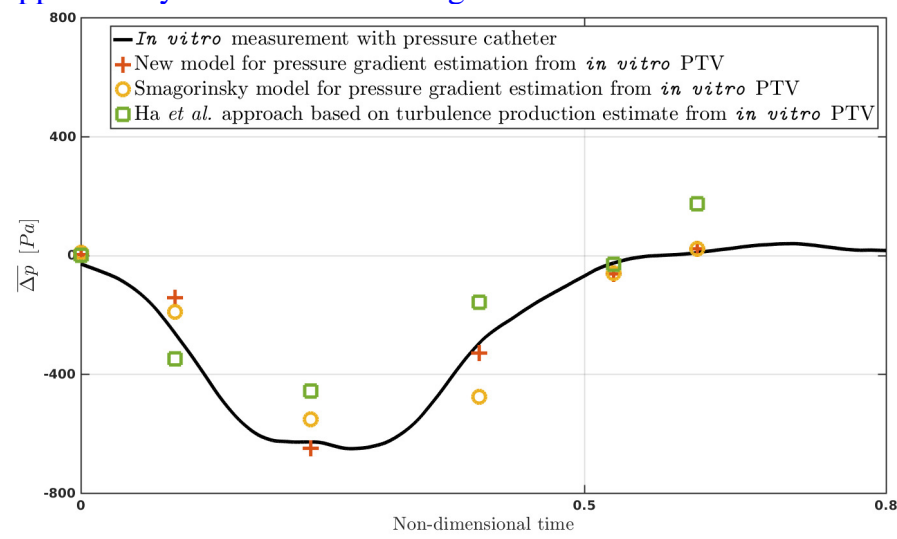

Fig. 8. Comparison of the time-averaged pressure difference measured with a pressure catheter during in vitro 3D-PTV experiments and the pressure difference estimated at six time instants during the pulse using the three models proposed in this study. Also shown are the estimates using the approach by Ha et al. [15], [16]

\section{LIMITATIONS}

Although our novel approach is based on a clear theoretical background and brings an improved and validated method for pressure drop estimation along the centreline as compared to the more general estimation methods when applied to in vitro 3D-PTV measurements, it also has some limitations. In fact, a subsequent step is still required to translate the new methods proposed here and based on subvoxel modelling to the clinical practice. In fact, given the recent advances in the 4D Flow magnetic resonance technique to assess ensembleaveraged velocities as well as the full Reynolds stress tensor [30], [31], it is foreseeable that the approach presented in this study will be applicable once a validated sequence for the evaluation of the first two terms of Eq. 19 and the ensembleaveraged cubed magnitude of the instantaneous rate-of-strain 
tensor will be available.

The cases treated in this study are limited to a single aorta geometry for which inflow conditions, wall compliance and the stenosis geometry and severity have been changed as part of PTV experiments. Applying our novel approach to more cases would enable to confirm the generality of the models for the accurate evaluation of both pressure gradient and SVS stress for disturbed aortic flows.

\section{CONCLUSION}

A novel approach for pressure gradient evaluation and subvoxel-scale stress estimation from under-resolved flow quantification has been validated against results from a direct numerical simulation as well as direct pressure catheter measurements. In addition to being validated for different sets of flow conditions (i.e. concentric and tricuspid severe stenoses, pulsatile and steady inflows, flexible and rigid walls), this approach offers the advantage of requiring only a limited number of points along the centreline of the aorta geometry to evaluate pressure drop. A Smagorinsky-based model for the estimation of SVS stress magnitude was optimised and was found to be more accurate yet more sensitive to errors due to variation in its composing terms than the estimation provided by the new model especially at a voxel size in the range between $1 \mathrm{~mm}$ to $750 \mu \mathrm{m}$. The new model was found to be more accurate for the evaluation of the projected pressure gradient than the Smagorinsky-based approximation and less sensitive to errors at a voxel size of $1 \mathrm{~mm}$ or less. Nonetheless, through the application of the proposed models to a pulsatile in vitro PTV case at a voxel size of $1.5 \mathrm{~mm}$, it has been noticed that the estimation of the pressure difference over six time instances over the pulse gave an error reduction of $21.5 \%$ with the Smagorinsky model and 36\% with the new model compared to the pressure difference obtained using the approach by $\mathrm{Ha}$ et al. [15], [16]. It is noteworthy that the latter approach gave the smallest error (around 30\% underestimation) among the more general methods for the irreversible pressure drop estimation for a steady case when compared to the value obtained from DNS and from our novel approach. Although this study focuses on the 3D-particle tracking velocimetry technique, the approach and models suggested can be applied to other techniques for flow investigation such as tomographic particle image velocimetry or large-eddy simulation.

\section{REFERENCES}

[1] J. C. Agüí and J. Jiménez, "On the performance of particle tracking," J. Fluid Mech., vol. 185, pp. 447-468, 1987.

[2] A. Altman and J. Gondzio, "Regularized symmetric indefinite systems in interior point methods for linear and quadratic optimization," Optimization Methods and Software, vol. 11, no. 1-4, pp. 275-302, 1999.

[3] H. Baumgartner et al., "Echocardiographic assessment of valve stenosis: EAE/ASE recommendations for clinical practice," J. Am. Soc. Echocardiogr., vol. 22, no. 1, pp. 1-23, 2009.

[4] O. Bech-Hanssen et al., "Assessment of effective orifice area of prosthetic aorticvalves with Doppler echocardiography: An in vivo and in vitro study," The Journal of Thoracic and Cardiovascular Surgery, vol. 122, no. 2, pp. 287-295, 2001.

[5] G.. Berkooz et al., "The proper orthogonal decomposition in the analysis of turbulent flows," Annu. Rev. Fluid Mech., vol. 25, pp. 539-575, 1993.
[6] M. Buzzicotti et al., "Effect of filter type on the statistics of energy transfer between resolved and subfilter scales from a-priori analysis of direct numerical simulations of isotropic turbulence," J. Turb., vol. 19, no. 2, pp 167-197, 2018.

[7] V. M. Canuto and Y. Cheng, "Determination of the Smagorinsky-Lilly constant C s, , Phys. Fluids, vol. 9, no. 5, pp 1368-1377, 1997.

[8] S. G. Chumakov, "Scaling properties of subgrid-scale energy dissipation," Phys. Fluids, vol. 19, no. 5, 2007.

[9] P. Corso et al., "Comprehensive In Vitro Study of the Flow Past Two Transcatheter Aortic Valves: Comparison with a Severe Stenotic Case," Ann. Biomed. Eng., vol. 47, no. 11, pp. 2241-2257, 2019.

[10] M. D. De Tullio et al., "Direct numerical simulation of the pulsatile flow through an aortic bileaflet mechanical heart valve," J. Fluid Mech. vol. 622, no. 11, pp. 259-290, 2009.

[11] M. Deville et al., "High-Order Methods for Incompressible Fluid Flow," Cambridge Monographs on Applied and Computational Mathematics, Cambridge: Cambridge University Press, 2002.

[12] A. Falahatpisheh et al., "Simplified Bernoulli's Method SignificantlyUnderestimates Pulmonary TransvalvularPressure Drop,", J. Magn. Res. Imaging, 2016.

[13] D. Garcia et al., "Assessment of Aortic Valve Stenosis Severity - A New Index Based on the Energy Loss Concept," Circulation, vol. 101, no. 7 , pp. 765-771, 2000.

[14] U. Gülan et al., "Shear-scaling-based approach for irreversible energy loss estimation in stenotic aorta flow - An in vitro study," J. Biomech., vol. 56, no. 11, pp. 89-96, 2017.

[15] H. Ha et al., "Estimating the irreversible pressure drop across a stenosis by quantifying turbulence production using 4D Flow MRI,' Sci. Rep., vol. 7, 46618, 2017.

[16] H. Ha et al., "Validation of pressure drop assessment using 4D flow MRI-based turbulence production in various shapes of stenoses," Magn. Res. in Medicine, vol. 81, 893-906, 2018.

[17] H. Hatoum et al., "Modeling of the Instantaneous Transvalvular Pressure Gradient in Aortic Stenosis," Ann. Biomed. Eng., vol. 47, no. 8, pp. $1748-1763,2019$

[18] J. C. R. Hunt et al., "Eddies, Streams, Convergence Zones in Turbulent Flows," Proceedings of the Summer Program in its Studying Turbulence Using Numerical Simulation Databases, Stanford, USA, pp. 193-208, 1988.

[19] V. Knobloch et al., "Mapping mean and fluctuating velocities by Bayesian multipoint MR velocity encoding-validation against 3D particle tracking velocimetry," Magnetic Resonance in Medicine, vol. 71, no. 4, pp. 1405-1415, 2014.

[20] S. E. Lee et al., "Direct numerical simulation of transitional flow in a stenosed carotid bifurcation," J. Biomech., vol.41, no. 11, pp. 2551-2561, 2008.

[21] NEK5000 v17.0. 2017. Argonne National Laboratory, Illinois. Available: https://nek5000.mcs.anl.gov.

[22] C. M. Otto, "Valvular Aortic Stenosis - Disease Severity and Timing of Intervention," J. Am. C. Card., vol.47, no. 11, 2006.

[23] S.-H. Peng and L. Davidson, "Approximation of subgrid-scale stresses based on the Leonard expansion," Proceedings of the $6^{\text {th }}$ Symposium on Turbulence, Heat and Mass Transfer, Rome, Italy, 2009.

[24] M. Piccinelli et al., "A Framework for Geometric Analysis of Vascular Structures: Application to Cerebral Aneurysms," IEE Trans. Med. Imag., vol. 28 , no. 8, 2009.

[25] S. Pope, "Large-eddy simulation," Turbulent Flows, Cambridge: Cambridge University Press, pp. 558-640, 2000.

[26] N. Quinlan, "Mechanical Loading of Blood Cells in Turbulent Flow," Computational Biomechanics for Medicine, Springer, pp. 1-13, 2014.

[27] F. A. Rajput and R. Zeltser, "Aortic Valve Replacement," StatPearls, Treasure Island (FL), 2019. [Online]. Available: https://www.ncbi.nlm.nih.gov/books/NBK537136/

[28] H. Rijsterborgh and J. Roelandt, "Doppler assessment of aortic stenosis: Bernoulli revisited," Ultrasound in Medicine and Biology, vol. 13, no. 5, 1987.

[29] S. S. Varghese et al., "Direct numerical simulation of stenotic flows, Part 1: Steady flow," J. Fluid Mech., vol. 582, pp. 553-580, 2007.

[30] V .Vishnevskiy V, et al.. "Deep variational network for rapid 4D flow MRI reconstruction," Nature Machine Intelligence, vol. 2., pp. 228-235, 2020.

[31] J. Walheim et al., "5D Flow Tensor MRI to Efficiently Map Reynolds Stresses of Aortic Blood Flow In-Vivo," Sci. Rep., vol. 9, 2019. 